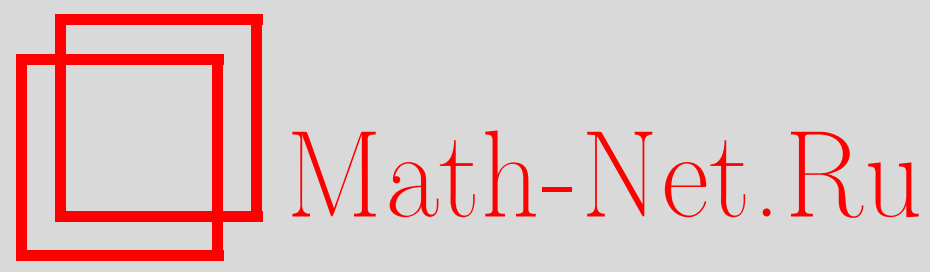

Е. В. Соколов, Финитная аппроксимируемость относительно сопряженности нисходящих HNN-расширений конечно порожденных абелевых групп, Матем. заметки, 2005, том 78, выпуск 5, 748-762

DOI: https://doi.org/10.4213/mzm2638

Использование Общероссийского математического портала Math-Net.Ru подразумевает, что вы прочитали и согласны с пользовательским соглашением http://www. mathnet.ru/rus/agreement

Параметры загрузки:

IP : 3.85 .73 .92

26 апреля 2023 г., 12:54:07

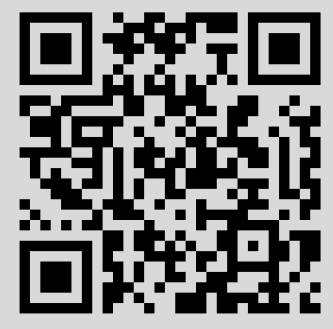




\title{
ФИНИТНАЯ АППРОКСИМИРУЕМОСТЬ ОТНОСИТЕЛЬНО СОПРЯЖЕННОСТИ НИСХОДЯЩИХ НNN-РАСШИРЕНИЙ КОНЕЧНО ПОРОЖДЕННЫХ АБЕЛЕВЫХ ГРУПП
}

\section{Е.В. Соколов}

\begin{abstract}
Доказано, что произвольное нисходящее $\mathrm{HNN}$-расширение конечно порожденной абелевой группы финитно аппроксимируемо относительно сопряженности.

Библиографоия: 14 названий.
\end{abstract}

1. Введение. Напомним, что если $A$ - некоторая группа и $\varphi$ - ее инъективный эндоморфизм, то нисходящим HNN-расширением группы $A$ с проходной буквой $t$ называется группа

$$
G=\left\langle A, t ; t^{-1} A t=A \varphi\right\rangle
$$

которая в системе порождающих, состоящей из всех образующих групшы $A$ и элемента $t$, определяется соотношениями групшы $A$ и всеми соотношениями вида $t^{-1} a t=a \varphi$, где $a \in A$ (введенные обозначения далее предполагаются фиксированньми).

Напомним также, что група $F$ назьвается финитно аппроксимируемой относительно предиката $\rho$, если для любых элементов и множеств элементов из $F$, не находящихся в отношении $\rho$, существует гомоморфизм групшы $F$ на конечную группу, при котором образы этих элементов и множеств по-прежнему не состоят в отношении $\rho$. Если $\rho$ представляет собой отношение равенства двух элементов, то упоминание о нем обычно опускают и говорят просто о финитной апшроксимируемости грушы $F$.

Нисходящее HNN-расширение представляет собой частный случай расширения Хигмана-Нейман-Неймана, возникающий, если хотя бы одна из связанных подгрупш совпадает с базовой групой. Специфика этого случая объясняется тем, что доказательства значительного числа результатов о произвольных HNN-расширениях существенным образом используют различие базовой группы и связанных подгрупп (см., например, [1]-[4]), так что непосредственное их применение к конструкциям данного типа невозможно. В то же время для нисходящих HNN-расширений решение ряда вопросов приобретает более законченный вид, как показьвают, например, работы [5] и [6].

В настоящей статье рассматривается ситуация, когда базовая группа $A$ является конечно порожденной абелевой. В этом случае HNN-расширение $G$ оказьвается метабелевой группой и потому его финитная аппроксимируемость следует из известной теоремы $\Phi$. Холла. Поскольку для HNN-расширений конечно порожденных абелевых групп, не являющихся нисходящими, обычная финитная аппроксимируемость равносильна свойству финитной апшроксимируемости относительно сопряженности (ФАС) 
$[2],[4]$, естественно предположить, что $G$ будет и $\Phi$ АС-группой. Однако подтвердить это предположение, получив соответствующий результат для метабелевых груп, невозможно ввиду контрпримера, построенного М.И. Каргаполовым и Е. И. Тимошенко в [7].

С другой стороны, если эндоморфизм $\varphi$ групшы $A$ в действительности является автоморфизмом, то HNN-расширение $G$ оказьвается обычньм расшепляющимся расширением групшы $A$ при помощи бесконечной циклической групшы с порождающим $t$. В случае конечно порожденной абелевой групшы $A$ это означает, что $G$ - полищиклическая группа, финитно аппроксимируемая относительно сопряженности согласно результата В.Н. Ремесленникова [8].

О.Е. Сенкевич [9] показал также, что HNN-расширение $G$ обладает свойством $\Phi$ АС, если базовая группа $A$ является свободной абелевой и содержит такую систему свободных порождающих $a_{1}, a_{2}, \ldots, a_{n}$, что $a_{i} \varphi=a_{i}^{k_{i}}$ для некоторых целых чисел $k_{i}$, отличных от нуля. Частный случай этого утверждения для циклической группы $A$ был доказан ранее в [10].

Настоящая статья продолжает до некоторой степени работу Сенкевича, и основной ее результат вьглядит следующим образом.

ТЕоремА. Произвольное нисходящее HNN-расширение конечно порожденной абелевой группы финитно аппроксимируемо относительно сопряженности.

Ввиду общего замечания, сделанного А.И. Мальцевым в [11], из сформулированной теоремы вытекает, в частности, что произвольное нисходящее HNN-расширение конечно порожденной абелевой групш имеет разрешимую проблему сопряженности. Необходимо отметить, однако, что этот результат получен ранее Г. А. Носковым [12] другими методами.

2. Предварительные замечания. Прежде всего введем несколько определений, необходимых для дальнейшего изложения.

Пусть $A$ - конечно порожденная абелева группа и $\varphi$ - ее инъективный эндоморфизм (данные обозначения предполагаются фиксированньми до конца раздела). Будем говорить, что элементы $a, b \in A \varphi$-әквивалентны, записывая этот факт в виде $a \sim_{\varphi} b$, если существуют целые неотрицательные числа $m$ и $n$ такие, что $a \varphi^{m}=b \varphi^{n}$.

Очевидно, что введенное отношение действительно является эквивалентностью. Класс всех элементов групшы $A, \varphi$-эквивалентных некоторому фиксированному элементу $a \in A$, будем далее обозначать через $a \Phi$. Поскольку эндоморфизм $\varphi$ является инъективньм, произвольный элемент множества $a \Phi$ либо совпадает с образом элемента $a$ относительно некоторой степени отображения $\varphi$, либо сам переходит в $a$ под действием эндоморфизма $\varphi^{n}$ для подходящего $n$. Поэтому множество $a \Phi$ естественно назвать $\varphi$-орбитой элемента $a$.

Подгруппу $B$ группы $A$ назовем $\varphi$-допустимой, если $B \varphi \leqslant B$, и $\varphi$-изолированной, если для каждого элемента $a \in A$ из $a \varphi \in B$ следует, что $a \in B$. Семейство всех $\varphi$-допустимых $\varphi$-изолированных подгрупп групшы $A$ будем обозначать символом $\Omega_{\varphi}(A)$. Кроме того, через $\Omega_{\varphi}^{f}(A)$ обозначим семейство всех подгрупп из $\Omega_{\varphi}(A)$, имеющих конечный индекс в группе $A$.

Заметим, что если подгруппа $B \leqslant A$-допустима, то эндоморфизм $\varphi$ индуцирует эндоморфизм факторгруппы $A / B$ (переводящий смежный класс $a B$ в смежньй класс 
$(a \varphi) B)$, которьй мы будем обозначать через $\varphi / B$. При этом $\varphi$-допустимость подгруппы $B$ является не только достаточным, но и необходимым условием корректности определения данного эндоморфизма.

Заметим также, что $\varphi$-допустимая подгруппа $B$ является $\varphi$-изолированной, т.е. принадлежит семейству $\Omega_{\varphi}(A)$ тогда и только тогда, когда эндоморфизм $\bar{\varphi}=\varphi / B$ инъективен. Поэтому для каждой подгрупшы $B \in \Omega_{\varphi}(A)$ мы можем определить на факторгруппе $A / B$ отношение $\bar{\varphi}$-эквивалентности, а на группе $A$ - отношение $\varphi$-эквивалентности по модулю $B$.

Более точно, будем говорить, что элементы $a, b \in A$-эквивалентны по модулю nодгруппы $B \in \Omega_{\varphi}(A)$ и писать $a \sim_{\varphi} b(\bmod B)$, если существуют целые неотрицательные числа $m$ и $n$ такие, что $a \varphi^{m} \equiv b \varphi^{n}(\bmod B)$.

Наконец, следуя общему определению, назовем группу $A$ финитно аппроксимируемой относительно $\varphi$-әквивалентности (или сокращенно $\Phi$ А $\varphi$-группой), если имеет место утверждение

$$
\forall a, b \in A\left(a \nsim_{\varphi} b \Longrightarrow \exists B \in \Omega_{\varphi}^{f}(A) a \nsim_{\varphi} b(\bmod B)\right)
$$

Будем говорить также, что группа $A$ регулярно финитно аппроксимируема относительно $\varphi$-әквивалентности (короче, является Р $Ф$ А $\varphi$-группой), если $A-\Phi А \varphi$-группа и для любых неединичного элемента $a \in A$ и натурального числа $n$ можно указать такую подгрупшу $B \in \Omega_{\varphi}(A)$, что индуцированньй эндоморфизм $\bar{\varphi}=\varphi / B$ факторгруппы $A / B$ является автоморфизмом конечного порядка, кратного $n$, и мощность $\bar{\varphi}$-орбиты элемента $a B$ совпадает с порядком автоморфизма $\bar{\varphi}$.

Следующее утверждение, принадлежащее Сенкевичу, объясняет, какое отношение понятие финитной аппроксимируемости относительно $\varphi$-эквивалентности имеет к рассматриваемой задаче.

ПРЕДЛОЖЕНИЕ 1. Нисходящее HNN-расширение $G=\left\langle A, t ; t^{-1} A t=A \varphi\right\rangle$ әруппь А финитно аппроксимируемо относительно сопряженности тогда и только тогда, когда $A$ является $\Phi$ А $\varphi$-группой.

ДокАЗАТЕЛЬСтво. Проверим сначала достаточность. Пусть $g$ и $h$ - произвольные несопряженные элементы группы $G$. Нам нужно лиш указать гомоморфизм группы $G$ на полициклическую групу, при котором классы сопряженности элементов $g$ и $h$ остаются различньми. Согласно упоминавшемуся результату В. Н. Ремесленникова [8] его всегда можно будет продолжить до гомоморфизма на конечную групу, сохраняющего это различие.

Из леммы Бриттона для произвольных HNN-расширений (см., например, [13, гл. IV, $\S 2])$ легко следует, что каждый элемент групшы $G$ однозначно представим в виде $t^{m} a t^{-n}$, где $a \in A, m, n$-целые неотрицательные числа и, если $m>0$ и $n>0$, то $a \notin A \varphi$. Поэтому, заменяя $g$ и $h$ подходящими сопряженньми с ними элементами, мы можем считать, что $g=t^{k} a$ и $h=t^{l} b$ для некоторых $k, l \in \mathbb{Z}, a, b \in A$. Более того, поскольку сопряженность элементов $g$ и $h$ равносильна сопряженности элементов $g^{-1}$ и $h^{-1}$, можно предполагать также, что $k \geqslant 0$.

Если $k \neq l$, то искомым будет естественный гомоморфизм группы $G$ на ее циклическую факторгруппу по нормальному замыканию подгрупшы $A$. Образы элементов $g$ и $h$ относительно этого гомоморфизма, очевидно, различны и, следовательно, несопряжены. 
Пусть $k=l$. Положим $A_{k}=\left\{c \varphi^{k} c^{-1} \mid c \in A\right\}$. Легко видеть, что для любого неотрицательного $k$ множество $A_{k}$ является подгруппй и принадлежит семейству $\Omega_{\varphi}(A)$. Докажем теперь одно вспомогательное утверждение.

Лемма. Элементы $u=t^{q}$ с $u v=t^{q} d$ группы $G$ сопряжены в этой группе тогда и только тогда, когда $c \sim_{\varphi} d\left(\bmod A_{q}\right)$.

ДокАЗАТЕЛьСтво. В самом деле, если $u=w^{-1} v w$, то, записьвая элемент $w$ в виде $t^{m} f t^{-n}$ для подходящих неотрицательных чисел $m, n$ и элемента $f \in A$, мы получаем соотношение $t^{-q} f t^{q} t^{-n} c t^{n}=t^{-m} d t^{m} f$, равносильное соотношению $f \varphi^{q} c \varphi^{n}=d \varphi^{m} f$, которое в свою очередь означает, что $c \sim_{\varphi} d\left(\bmod A_{q}\right)$.

Таким образом, поскольку элементы $g$ и $h$ не сопряжены в групе $G, a \nsim_{\varphi} b\left(\bmod A_{k}\right)$.

Рассмотрим теперь отдельно ситуацию, когда $k=0$. В этом случае согласно лемме элементы $a$ и $b$ не являются $\varphi$-эквивалентньми в группе $A$ и, так как последняя представляет собой $\Phi$ А $\varphi$-групу, существует подгруппа $B \in \Omega_{\varphi}^{f}(A)$ такая, что $a \nsim_{\varphi} b$ $(\bmod B)$. Обозначим через $q$ порядок индуцированного автоморфизма $\varphi / B$ (конечной) факторгруппы $A / B$. Тогда, очевидно, $A_{q} \leqslant B$ и, стало быть, $a \nsim_{\varphi} b\left(\bmod A_{q}\right)$.

Пусть группа $\bar{G}$ представляет собой нисходящее $\mathrm{HNN}$-расширение $\left\langle\bar{A}, \tau ; \tau^{-1} \bar{A} \tau=\right.$ $\bar{A} \bar{\varphi}\rangle$, где $\bar{A}=A / A_{k}$, если $k>0, \bar{A}=A / A_{q}$, если $k=0$, и $\bar{\varphi}-$ эндоморфизм, индуцированньй в факторгруппе $\bar{A}$ эндоморфизмом $\varphi$. Тогда при отображении $\rho: G \rightarrow \bar{G}$, продолжающем естественньй гомоморфизм $\varepsilon: A \rightarrow \bar{A}$ и переводящем элемент $t$ в $\tau$, все определяюшие соотношения групшы $G$ переходят в равенства, справедливые в групе $\bar{G}$. Поэтому отображение $\rho$, в свою очередь, может быть продолжено до гомоморфизма на группу $\bar{G}$, которьй мы будем обозначать той же буквой. Покажем, что этот гомоморфизм и является искомым.

$\mathrm{B}$ самом деле, в силу соотношений $a \nsim_{\varphi} b\left(\bmod A_{k}\right)($ при $k \neq 0)$ и $a \nsim_{\varphi} b \bmod A_{q}$ $($ при $k=0) \bar{\varphi}$-орбиты элементов $a \rho$ и $b \rho$ в группе $\bar{A}$ не совпадают. Поскольку и при $k=0$, и при $k \neq 0 \bar{A}_{k}=1$, отсюда следует, что $a \rho \nsim_{\bar{\varphi}} b \rho\left(\bmod \bar{A}_{k}\right)$ и, стало быть, согласно лемме элементы $g \rho$ и $h \rho$ не сопряжены в групе $\bar{G}$. Остается лишь вспомнить, что при любом $k$ эндоморфизм $\bar{\varphi}$ является автоморфизмом конечного порядкагруппы $\bar{A}$ и потому $\bar{G}$ - полищиклическая група. Тем самым, доказательство достаточности закончено.

Необходимость условия предложения с учетом отмеченного вьше проверяется совсем легко.

Пусть $a$ и $b$ - произвольные элементы групшы $A$, не являющиеся $\varphi$-эквивалентными. Согласно лемме эти элементы не сопряжены в групше $G$ и в силу свойства $\Phi$ АС продолжают оставаться таковыми в некотором конечном гомоморфном образе $\bar{G}=G / H$ группы $G$. Отображение $\bar{\varphi}$ подгруппы $\bar{A}=A H / H$ в себя, переводящее элемент $a H$ в $(a \varphi) H$, совпадает, очевидно, с автоморфизмом этой подгрупшы, порожденным сопряжением при помощи элемента $t H$. В частности, оно корректно определено и инъективно, и это означает, что ядро $B$ гомоморфизма группы $A$, получающегося ограничением на $A$ естественного гомоморфизма $\varepsilon: G \rightarrow \bar{G}$, принадлежит семейству $\Omega_{\varphi}^{f}(A)$. Остается заметить, что $\bar{\varphi}$-орбиты элементов $a H$ и $b H$ совпадают с их классами сопряженности в группе $\bar{G}$ и потому различны. Таким образом, $a \nsim_{\varphi} b(\bmod B)$.

Из предложения 1 и финитной аппроксимируемости относительно сопряженности произвольной полициклической группы сразу же вытекает 
ПРЕДЛОЖЕНИЕ 2. Если эндоморфизм $\varphi$ является автоморфизмом, то группа $A$ финитно аппроксимируема относительно ч-әквивалентности.

Следующее утверждение показьвает, что свойства $\Phi$ А $\varphi$ и Р $\Phi$ А являются наследственньми, а также, с учетом некоторых ограничений, сохраняются при переходе к конечньм расширениям. Поскольку далее речь будет идти исключительно об абелевых группах и векторных пространствах, мы перейдем к аддитивной записи групповой операции.

ПРЕДЛОЖЕНИЕ 3. Пусть $A_{1}-\varphi$-допустимая nодгрynna гpynnbl $A$ u $\varphi_{1}$ - ограничение әндоморфизма $\varphi$ на подгруппу $A_{1}$. Тогда

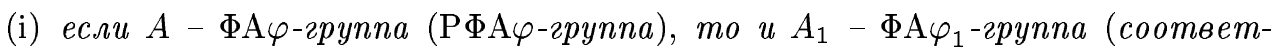
ственно РФА $\varphi_{1}$-группа);

(ii) если группа $A$ - без кручения, а подәруппа $A_{1}$ имеет конечный индекс в $A$ и является $\Phi \mathrm{A} \varphi_{1}$-группой (РФА $\varphi_{1}$-группой), то и вся группа $A$ обладает соответствующим свойством.

ДокАЗАТЕЛЬСТво. (i) Пусть сначала $a$ и $b$ - произвольные элементы подгрупшы $A_{1}$, удовлетворяющие условию $a \nsim_{\varphi_{1}} b$. Так как $\varphi_{1}$ является ограничением эндоморфизма $\varphi$, то, очевидно, $a \nsim_{\varphi} b$ и в силу финитной аппроксимируемости группы $A$ относительно $\varphi$-эквивалентности существует такая подгруппа $B \in \Omega_{\varphi}^{f}(A)$, что $a \nsim_{\varphi} b(\bmod B)$.

Положим $B_{1}=B \cap A_{1}$. Поскольку обе подгрупшы $B$ и $A_{1}$ являются $\varphi$-допустимыми, этим свойством обладает и подгруппа $B_{1}$. Очевидно также, что она $\varphi$-изолирована в группе $A_{1}$. Таким образом, $B_{1} \in \Omega_{\varphi_{1}}^{f}\left(A_{1}\right)$ и $a \nsim_{\varphi_{1}} b\left(\bmod B_{1}\right)$ ввиду включения $B_{1} \leqslant B$.

Предположим теперь, что $A$ является Р $\Phi$ А $\varphi$-группй и $a-$ произвольньй ненулевой элемент подгрупшы $A_{1}, n$ - натуральное число. Как и выше, выберем такую подгрупу $B \in \Omega_{\varphi}(A)$, что индуцированньй эндоморфизм $\bar{\varphi}=\varphi / B$ факторгрупшы $A / B$ оказывается автоморфизмом, порядок $q$ которого конечен и удовлетворяет условиям $n \mid q$ и $(a+B) \bar{\varphi}^{k} \neq a+B$ при $0<k<q$. Полагая $B_{1}=B \cap A_{1}$, мы видим, что с точностью до изоморфизма факторгрупп $\left(A_{1}+B\right) / B$ и $A_{1} / B_{1}$ автоморфизм $\bar{\varphi}_{1}=\varphi_{1} / B_{1}$ последней является сужением автоморфизма $\bar{\varphi}$. Поэтому его порядок оказывается ограниченным с одной стороны порядком $q$ автоморфизма $\bar{\varphi}$, а с другой - мощностью $\bar{\varphi}_{1}$-орбиты элемента $a+B_{1}$, которая также не меньше $q$ ввиду включения $B_{1}$ в $B$. Таким образом, $\left|\bar{\varphi}_{1}\right|=q$ и подгрупша $B_{1}$ является искомой.

(ii) Пусть $n=\left[A: A_{1}\right]$ и $\rho$ - гомоморфизм группы $A$, переводящий каждьй элемент $a \in A$ в элемент $n a$. Так как групша $A$ без кручения, отображение $\rho$ задает изоморфизм этой групшы на подгруппу $A_{2}=n A \leqslant A_{1}$. Полагая $\varphi^{\rho}=\rho^{-1} \varphi \rho$, мы переносим в соответствии с этим изоморфизмом эндоморфизм $\varphi$ в группу $A_{2}$. Поэтому (регулярная) финитная аппроксимируемость группы $A$ относительно $\varphi$-эквивалентности оказьвается равносильной свойству $\Phi$ А $\varphi^{\rho}$ (соответственно, $\left.\mathrm{P} \Phi \mathrm{A} \varphi^{\rho}\right)$ группы $A_{2}$.

Заметим теперь, что ввиду перестановочности отображений $\rho$ и $\varphi$ на элементах подгруппы $A_{2}$ эндоморфизм $\varphi^{\rho}$ в действительности является сужением эндоморфизма $\varphi$. Так как к тому же $A_{2} \leqslant A_{1}$, отображение $\varphi^{\rho}$ можно рассматривать и как ограничение на групш $A_{2}$ эндоморфизма $\varphi_{1}$. Стало быть, для доказательства необходимых свойств группы $A_{2}$ мы можем воспользоваться первой частью настоящего предложения.

Следующее утверждение позволяет несколько упростить проверку свойства финитной аппроксимируемости относительно $\varphi$-эквивалентности, снимая требование конеч- 
ности индекса подгруппы $B$, по модулю которой элементы должны оставаться неэквивалентными.

ПРЕДЛОЖЕНИЕ 4. Группа $A$ финитно аппроксимируема относительно $\varphi$-эквивалентности тогда и только тогда, когда для любых әлементов $a, b \in A, a \nsim_{\varphi} b$, существует такая подгруппа $B \in \Omega_{\varphi}(A)$, что $a \nsim_{\varphi} b(\bmod B)$ и мощности орбит әлементов $a+B u b+B$ факторгруппь $A / B$ относительно индуцированного эндоморфизма $\varphi / B$ конечны.

ДокАЗАТЕЛЬСТво. Необходимость данного утверждения очевидна, проверим достаТОчность.

Пусть $a$ и $b$ - произвольные элементы грушшы $A$, не являюшиеся $\varphi$-эквивалентными. Ввиду условия мы можем сразу же считать их $\varphi$-орбиты конечными. Поэтому воспользуемся обычной финитной аппроксимируемостью группы $A$ и выберем в ней подгруппу $C$ конечного индекса, не содержащую ни одного элемента из множества $M=\{u-v \mid$ $u \in a \Phi, v \in b \Phi\}$ (для обоснования возможности такого выбора нужно еще заметить, что $a \Phi \cap b \Phi=\varnothing$ и, следовательно, $0 \notin M)$. Не ограничивая общности рассуждений, мы можем считать также, что подгруппа $C$ имеет вид $n A$ для некоторого подходящего натурального числа $n$ и, таким образом, является $\varphi$-допустимой.

Обозначим через $B$-изолятор подгрупшы $C$ в группе $A$, т.е. наименьшую $\varphi$-изолированную подгруппу группы $A$, содержащую $C$. Легко видеть, что эта подгруппа совпадает с множеством $\left\{a \in A \mid \exists n a \varphi^{n} \in C\right\}$ и, стало быть, принадлежит семейству $\Omega_{\varphi}^{f}(A)$. Так как $M \varphi \subseteq M$, отсюда следует также, что подгруппа $B$ по-прежнему не содержит ни одного элемента множества $M$. Поэтому $a \nsim_{\varphi} b(\bmod B)$, что и требовалось.

Перейдем теперь к доказательству основного утверждения данного раздела, которое, в частности, дает возможность сводить вопрос о финитной аппроксимируемости группы $A$ относительно $\varphi$-эквивалентности к изучению ее прямых слагаемых.

ПРЕДЛОЖЕНИЕ 5. Пусть $B$ - некоторая подгруппа из семейства $\Omega_{\varphi}(A), \varphi_{B}-$ ограничение эндоморфизма $\varphi$ на подгруппу $B$ и $\bar{\varphi}=\varphi / B$ - эндоморфизм факторгруппь $\bar{A}=A / B$, индуцированный отображением $\varphi$. Пусть также $\bar{A}$ является РФА̄̄-гpynпой. Тогда

(i) если $B-\Phi \mathrm{A} \varphi_{B}$-гpynna, mo $A-\Phi \mathrm{A} \varphi$-гpynna;

(ii) если $B-\mathrm{P} \Phi \mathrm{A} \varphi_{B}{ }^{-\imath p y n n a, ~ m o ~} A-\mathrm{P} \Phi \mathrm{A} \varphi$-групnа.

ДокАЗАтЕЛьСтво. (i) Пусть $a$ и $b$-произвольные неэквивалентные элементы группы $A$. С учетом предыдущего предложения нам достаточно указать подгрупу $F \in$ $\Omega_{\varphi}(A)$, по модулю которой $\varphi$-орбиты этих элементов конечны и не совпадают.

Обозначим через $\bar{a}$ и $\bar{b}$ образы элементов $a, b$ в группе $\bar{A}$ и рассмотрим три возможности.

1) $\bar{a}=\bar{b}=0$. В этом случае элементы $a$ и $b$ принадлежат подгрупе $B$. Воспользуемся ее апроксимируемостью и найдем такую подгрупу $F \in \Omega_{\varphi_{B}}^{f}(B)$, что $a \nsim_{\varphi_{B}} b$ $(\bmod F)$. Так как подгрупша $B$-изолирована в групе $A$, подгрупша $F$ также является $\varphi$-изолированной в $A$ и, стало быть, принадлежит семейству $\Omega_{\varphi}(A)$. Кроме того, отсюда следуют равенства $a \Phi=a \Phi_{B}$ и $b \Phi=b \Phi_{B}$. Поэтому мощности $\varphi$-орбит элементов $a$, $b$ по модулю $F$ конечны, и подгруппа $F$ оказьвается искомой.

2) Хотя бы один из элементов $\bar{a}, \bar{b}$ отличен от нуля и $\bar{a} \nsim_{\bar{\varphi}} \bar{b}$. Здесь достаточно использовать аппроксимируемость группы $\bar{A}$, указав подгруппу $\bar{F} \in \Omega_{\bar{\varphi}}^{f}(\bar{A})$, по модулю 
которой сохраняется соотношение $\bar{a} \nsim_{\bar{\varphi}} \bar{b}$. В качестве искомой подгрупшы $F$ теперь следует взять прообраз подгрупшы $\bar{F}$ относительно естественного гомоморфизма $\varepsilon: A \rightarrow \bar{A}$.

3 ) Хотя бы один из элементов $\bar{a}, \bar{b}$ отличен от нуля и $\bar{a} \sim_{\bar{\varphi}} \bar{b}$. Заменяя при необходимости элементы $a$ и $b$ их образами относительно подходящих степеней эндоморфизма $\varphi$, мы можем считать, что $\bar{a}=\bar{b} \neq 0$.

Положим $g=a-b$. Так как $a$ и $b$ неэквивалентны, этот элемент отличен от нуля и в силу сделанного предположения о равенстве элементов $\bar{a}$ и $\bar{b}$ принадлежит подгруппе $B$. Ввиду апшроксимируемости последней найдется подгруппа $D \in \Omega_{\varphi_{B}}^{f}(B) \subseteq \Omega_{\varphi}(A)$, не содержащая элемента $g$.

Предполагая, что для некоторых неотрицательных чисел $m$ и $n a \varphi^{m} \equiv b \varphi^{n}(\bmod D)$, и переходя к факторгруппе $\bar{A}$, мы получаем, что $\bar{b} \bar{\varphi}^{m}=\bar{a} \bar{\varphi}^{m}=\bar{b} \bar{\varphi}^{n}$ и в силу инъективности эндоморфизма $\bar{\varphi} \bar{b} \bar{\varphi}^{|m-n|}=\bar{b}$. Теперь следует заметить, что в регулярно финитно апроксимируемой групе, каковой является група $\bar{A}$, мощность орбиты произвольного отличного от нуля элемента делится на любое наперед заданное натуральное число и, следовательно, бесконечна. Таким образом, $m=n$ и $g \varphi^{n}=a \varphi^{m}-b \varphi^{n} \in D$. Но подгруппа $D$-изолирована, поэтому $g \in D$.

Полученное противоречие доказьвает, что $a \nsim_{\varphi} b(\bmod D)$ и, стало быть, мы с самого начала могли считать подгруппу $B$ конечной.

Обозначим через $r$ порядок (теперь уже заведомо автоморфизма) $\varphi_{B}$. Ввиду регулярной финитной апшроксимируемости группы $\bar{A}$ найдется такая подгруппа $\bar{C} \in \Omega_{\bar{\varphi}}^{f}(\bar{A})$, что эндоморфизм $\overline{\bar{\varphi}}=\bar{\varphi} / \bar{C}$ факторгрупшы $\bar{A} / \bar{C}$, индуцированньй отображением $\bar{\varphi}$, является автоморфизмом и его порядок $q$ конечен, делится на $r$ и совпадает с мощностью $\overline{\bar{\varphi}}$-орбиты элемента $\bar{b}+\bar{C}$.

Положим $F=\left\{f \varphi^{q}-f \mid f \in A\right\}$. Как уже было отмечено при доказательстве предложения 1 , множество $F$ является подгруппой и принадлежит семейству $\Omega_{\varphi}(A)$. Поскольку отображение $\bar{\varphi}^{q}$ по модулю подгруппы $\bar{C}$ является тождественньм, подгруппа $\bar{F}=(F+B) / B$ содержится в $\bar{C}$. Покажем, что $F \cap B=0$.

В самом деле, пусть $d$ - произвольный элемент подгруппы $F \cap B$. Запишем его в виде $d=f \varphi^{q}-f$ для подходящего элемента $f \in A$.

Так как $d \in B$ и $r$ делит $q$, отображение $\varphi^{q}$ действует на элемент $d$ тождественно. Поэтому, обозначая через $s$ порядок групшы $B$, мы получаем, что $f \varphi^{q s}=f+s d=f$. Напомним теперь, что в силу регулярной финитной аппроксимируемости групшы $\bar{A}$ все ненулевые $\bar{\varphi}$-орбиты в ней бесконечны. Следовательно, $f \in B$ и $d=f \varphi^{q}-f=0$.

Для завершения доказательства утверждения (i) нам осталось проверить, что элементы $a$ и $b$ не являются $\varphi$-эквивалентными по модулю подгруппы $F$.

Предположим противное: пусть для некоторых целых неотрищательных чисел $m$ и $n$ $a \varphi^{m}-b \varphi^{n} \in F$. Тогда $\bar{b} \bar{\varphi}^{m}-\bar{b} \bar{\varphi}^{n}=\bar{a} \bar{\varphi}^{m}-\bar{b} \bar{\varphi}^{n} \in \bar{F} \leqslant \bar{C}$ и, так как порядок $\bar{\varphi}$-орбиты элемента $\bar{b}$ по модулю подгруппы $\bar{C}$ равен $q$, то $m \equiv n(\bmod q)$. Отсюда следует, что $b \varphi^{m} \equiv b \varphi^{n}$ и $g \varphi^{m} \equiv a \varphi^{m}-b \varphi^{n} \equiv 0(\bmod F)$. Тем самым, мы получаем ненулевой элемент $g \varphi^{m}$, одновременно принадлежащий подгруппам $F$ и $B$, что противоречит доказанному вьше. Утверждение (i) доказано.

(ii) Финитная аппроксимируемость относительно $\varphi$-эквивалентности группы $A$ здесь обеспечивается частью (i), поэтому нам нужно лишь проверить справедливость дополнительного условия регулярности.

Пусть $a$-произвольный ненулевой элемент группы $A, n \in \mathbb{N}$. Если элемент $a$ не принадлежит подгрупше $B$, то образ его в группе $\bar{A}$ отличен от нуля и подгрупшу, по модулю 
которой эндоморфизм $\varphi$ и элемент $a$ будут обладать всеми необходимыми свойствами, можно найти, пользуясь регулярной финитной апроксимируемостью групшы $\bar{A}$.

Пусть $a \in B$. Так как група $B$ регулярно финитно аппроксимируема, мы можем считать, что $\varphi_{B}$ является автоморфизмом и его порядок $q$ конечен, делится на $n$ и совпадает с мощностью $\varphi_{B}$-орбиты элемента $a$.

Как и вьше, проверяется, что подгруппа $F=\left\{f \varphi^{q}-f \mid f \in A\right\} \in \Omega_{\varphi}(A)$ тривиально пересекается с $B$. Поэтому мошность фактормножества $(a \Phi+F) / F$ по-прежнему равна $q$ и, стало быть, совпадает с порядком индуцированного в факторгруппе $A / F$ автоморфизма $\varphi / F$. Это означает, что подгруппа $F$ является искомой.

3. Доказательство основной теоремы. Этот раздел, как и предыдуший, начнем с необходимых определений.

Пусть $A$ - свободная абелева группа конечного ранга и $\varphi$ - ее инъективный эндоморфизм. Если зафиксировать некоторую систему свободных порождающих групшы $A$, то эндоморфизму $\varphi$ по аналогии с обычным линейньм оператором конечномерного векторного пространства можно сопоставить невырожденную целочисленную матрищу $M_{\varphi}$. Характеристический многочлен $\chi_{\varphi}$ этой матрицы (не зависящий уже от выбора базиса группы $A$ ) назьвают характеристическим многочленом эндоморфизма $\varphi$.

Если отображение $\varphi$ в действительности является автоморфизмом, то матрища $M_{\varphi}^{-1}$ также оказывается целочисленной и, следовательно, свободный член $\chi_{0}$ многочлена $\chi_{\varphi}$ (совпадаюший с определителем матрицы $M_{\varphi}$ ) должен быть равен \pm 1 . Верно, разумеется, и обратное: если $\left|\chi_{0}\right|=1$, то $\varphi$ - автоморфизм группы $A$.

В связи с этим имеет смысл ввести специальный термин для обозначения целочисленного унитарного многочлена, свободньй член которого равен \pm 1 . Мы назовем такой многочлен сверхунитарным.

Далее, под минимальны.м многочленом комплексного алгебраического числа будем понимать (однозначно определенньй) унитарньй минимальный многочлен этого числа над полем $\mathbb{Q}$. Напомним, что алгебраическое число, минимальный многочлен которого имеет целые коэффициенты, назьвается иелым алгебраическим.

Если $c$ - некоторое ненулевое целое алгебраическое число, то через $Z_{c}$ мы будем обозначать аддитивную группу кольца $\mathbb{Z}[c]$, а через $\varphi_{c}-$ эндоморфизм этой групш, осуществляющий умножение каждого ее элемента на $c$. Поскольку при любом выборе числа $c$ в кольце $\mathbb{Z}[c]$ нет делителей нуля, эндоморфизм $\varphi_{c}$ всегда является инъективньм и мы можем сформулировать

ПРЕДЛОЖЕНИЕ 6. Пусть $с$ - ненулевое иелое алгебраическое число, минимальный многочлен которого не является сверхунитарным. Тогда $Z_{c}$ является $\mathrm{P} \Phi \mathrm{A} \varphi_{c}$-группой.

ДОКАЗАТЕЛЬСТВО. Пусть $g(x)=x^{q}-g_{q-1} x^{q-1}-\cdots-g_{1} x-g_{0}$ - минимальньй многочлен элемента $c$ и $\lambda_{1}, \lambda_{2}, \ldots, \lambda_{q}$ все его комплексные корни. Будем считать, что $\left|\lambda_{j}\right|>1$ при $1 \leqslant j \leqslant r,\left|\lambda_{j}\right|=1$ при $r+1 \leqslant j \leqslant r+s,\left|\lambda_{j}\right|<1$ при $r+s+1 \leqslant j \leqslant q$ и положим для удобства $\mu_{j}=\lambda_{r+j}, 1 \leqslant j \leqslant s, \nu_{j}=\lambda_{r+s+j}, 1 \leqslant j \leqslant t$, где $t=q-r-s$. Поскольку многочлен $g$ - целочисленный и не является сверхунитарным, $r \geqslant 1$, в то время как числа $s$ и $t$ могут быть равны нулю.

Из неприводимости многочлена $g$ (здесь и далее отношения делимости и неприводимости многочленов рассматриваются над кольцом $\mathbb{Z})$ следует, что элементы $c^{0}, c^{1}, \ldots$, 
$c^{q-1}$ составляют базис группы $Z_{c}$. Для каждого элемента $a \in Z_{c}$ через $M_{a}$ будем обозначать матрицу, которая соответствует в этом базисе эндоморфизму $\varphi_{a}$ (умножающему всякий элемент групшы $Z_{c}$ на $\left.a\right)$, и через $N(a)$ - модуль определителя матрицы $M_{a}$.

Поскольку при любом $a M_{a}$ - целочисленная матрища, значениями функции $N(\cdot)$ будут целые неотрицательные числа. При этом $N(a)=0$ тогда и только тогда, когда нулю равна некоторая нетривиальная линейная комбинация элементов $a, a c, \ldots, a c^{q-1}$ с целыми коэффициентами, что ввиду отсутствия в кольце $\mathbb{Z}[c]$ делителей нуля равносильно условию $a=0$. Легко видеть также, что для любых элементов $a, b \in Z_{c}$ $M_{a+b}=M_{a}+M_{b}$ и $M_{a b}=M_{a} M_{b}$. Поэтому, в частности, $N(a b)=N(a) N(b)$.

ЛЕмма 1. Для каждого многочлена $f \in \mathbb{Z}[x]$

$$
N(f(c))=\prod_{j=1}^{q}\left|f\left(\lambda_{j}\right)\right|
$$

ДокАЗАТЕЛЬСТво. Из отмеченных только что соотношений следует, что $N(f(c))=$ $\left|\operatorname{det} M_{f(c)}\right|=\left|\operatorname{det} f\left(M_{c}\right)\right|$. Так как для любой матрицы $T \in G L(q, \mathbb{C})$

$$
\operatorname{det} f\left(M_{c}\right)=\operatorname{det} T^{-1} f\left(M_{c}\right) T=\operatorname{det} f\left(T^{-1} M_{c} T\right)
$$

при вычислении числа $N(f(c))$ мы можем заменить $M_{c}$ эквивалентной ей матрицей $M_{c}^{\prime}$ в нормальной жордановой форме.

Заметим теперь, что матрица $M_{c}$ имеет вид

$$
\left(\begin{array}{ccccc}
0 & 1 & & & 0 \\
& 0 & \ddots & & \\
& & \ddots & 1 & \\
0 & & & 0 & 1 \\
g_{0} & g_{1} & \cdots & g_{q-2} & g_{q-1}
\end{array}\right)
$$

и характеристический многочлен, равный $g(x)$, поэтому все ее комплексные собственные значения совпадают с числами $\lambda_{j}, 1 \leqslant j \leqslant q$. Отсюда сразу же вытекает, что $f\left(M_{c}^{\prime}\right)$ - верхнетреугольная матрица, наглавной диагонали которой стоят числа $f\left(\lambda_{j}\right)$, и

$$
N(f(c))=\left|\operatorname{det} f\left(M_{c}^{\prime}\right)\right|=\prod_{j=1}^{q}\left|f\left(\lambda_{j}\right)\right| .
$$

Следующее утверждение проверяется при помощи совершенно элементарных рассуждений, поэтому мы ограничимся здесь лишь его формулировкой.

Лемма 2. Пусть $C_{1}=\{z \in \mathbb{C}|| z \mid=1\}$. Каково бы ни было натуральное число $s$, найдется положительное действительное число в, удовлетворяющее условию: $\forall z_{1}, z_{2}, \ldots, z_{s} \in C_{1} \backslash\{1\} \quad \exists m \in \mathbb{N} \quad \forall k, 1 \leqslant k \leqslant s,\left|z_{k}^{m}-1\right|>\varepsilon$. 
ЛЕмма 3. Каковы бы ни были натуральные числа $\xi$ и п, найдется такое четное $m \in \mathbb{N}$, что $n \mid m$ и для любых многочленов $u, v \in \mathbb{Z}[x]$ справедливо утверждение:

$$
\begin{aligned}
(\forall j, 1 & \left.\leqslant j \leqslant q,\left(\left|u\left(\lambda_{j}\right)\right|<\xi \wedge\left|v\left(\lambda_{j}\right)\right|<\xi\right)\right) \\
& \Longrightarrow\left(\forall k, 0 \leqslant k \leqslant \frac{m}{2}, N\left(u(c)-c^{k} v(c)\right)<N\left(c^{m}-1\right)\right) .
\end{aligned}
$$

ДоКАЗАТЕЛЬСтво. Если количество $s$ корней многочлена $g$, равных по модулю единище, отлично от нуля, воспользуемся леммой 2 и найдем соответствующее ему число $\varepsilon$, которое без потери обшности можно считать меньшим единицы, в противном случае положим $\varepsilon=1 / 2$.

Так как $\left|\nu_{j}\right|<1,1 \leqslant j \leqslant t$, то при всех $k$, начиная с некоторого $m_{1} \in \mathbb{N}$, справедливы неравенства $\left|\nu_{j}^{k}\right|<1-\varepsilon$. Точно так же для некоторого $m_{2} \in \mathbb{N}$

$$
\left|\lambda_{j}^{k}\right|>\left(\frac{2 \xi}{\varepsilon}\right)^{q+1}
$$

при $1 \leqslant j \leqslant r$ и $k \geqslant m_{2}$.

Поскольку многочлен $g$ неприводим и не является сверхунитарньм, он взаимно прост с многочленом $x^{2 m_{1} m_{2} n}-1$. Поэтому все числа $z_{j}=\mu_{j}^{2 m_{1} m_{2} n}, 1 \leqslant j \leqslant s$, отличны от единицы и, применяя к ним лемму 2 , мы можем найти такое число $m_{3} \in \mathbb{N}$, что для каждого $j\left|z_{j}^{m_{3}}-1\right|>\varepsilon$.

Положим $m=2 m_{1} m_{2} m_{3} n$. Тогда в силу леммы 1

$$
\begin{aligned}
N\left(c^{m}-1\right) & =\prod_{j=1}^{q}\left|\lambda_{j}^{m}-1\right| \geqslant \prod_{j=1}^{r}\left(\left|\lambda_{j}^{m}\right|-1\right) \cdot \prod_{j=1}^{s}\left|\mu_{j}^{m}-1\right| \cdot \prod_{j=1}^{t}\left(1-\left|\nu_{j}^{m}\right|\right) \\
& >\varepsilon^{s+t} \prod_{j=1}^{r}\left(\left|\lambda_{j}^{m}\right|-1\right) .
\end{aligned}
$$

С другой стороны, для произвольных многочленов $u, v \in \mathbb{Z}[x]$, удовлетворяющих необходимьм неравенствам,

$$
N\left(u(c)-c^{k} v(c)\right) \leqslant \prod_{j=1}^{q}\left(\left|u\left(\lambda_{j}\right)\right|+\left|\lambda_{j}^{k}\right|\left|v\left(\lambda_{j}\right)\right|\right)<\xi^{q} \prod_{j=1}^{q}\left(\left|\lambda_{j}^{k}\right|+1\right)<2^{s+t} \xi^{q} \prod_{j=1}^{r}\left(\left|\lambda_{j}^{k}\right|+1\right) .
$$

Так как $m / 2 \geqslant m_{2}$, то для каждого $j, 1 \leqslant j \leqslant r$,

$$
\varepsilon^{q}\left(\left|\lambda_{j}^{m / 2}\right|-1\right)>(2 \xi)^{q}, \quad \varepsilon^{q}\left(\left|\lambda_{j}^{m}\right|-1\right)>(2 \xi)^{q}\left(\left|\lambda_{j}^{m / 2}\right|+1\right)
$$

и при $k \leqslant m / 2$

$$
\varepsilon^{q}\left(\left|\lambda_{j}^{m}\right|-1\right)>(2 \xi)^{q}\left(\left|\lambda_{j}^{k}\right|+1\right) .
$$

Перемножая $r$ последних неравенств и учитывая, что $\varepsilon<1 \leqslant \xi$, мы получаем соотношения $N\left(u(c)-c^{k} v(c)\right)<N\left(c^{m}-1\right), 0 \leqslant k \leqslant m / 2$, что и требовалось. 
Пусть теперь $a, b \in Z_{c}$ - произвольные элементы, не являющиеся $\varphi_{c}$-эквивалентными, и $n$ - некоторое натуральное число. Не ограничивая общности рассуждений, мы можем предположить, что $b \neq 0$.

Напомним, что при любом выборе числа $m \in \mathbb{N}$ подгруппа $B_{m}=\left(c^{m}-1\right) Z_{c}=$ $\left\{d \varphi_{c}^{m}-d \mid d \in Z_{c}\right\}$ принадлежит семейству $\Omega_{\varphi_{c}}\left(Z_{c}\right)$ и отображение $\bar{\varphi}_{c}=\varphi_{c} / B_{m}$ факторгруппы $Z_{c} / B_{m}$ является автоморфизмом этой группы порядка $m$. Поэтому для доказательства свойства $\Phi$ А $\varphi_{c}$ групшы $Z_{c}$ нам в силу предложения 4 достаточно найти такое $m$, при котором элементы $a$ и $b$ не будут $\varphi_{c}$-эквивалентными по модулю подгруппы $B_{m}$. Если же $m$ удастся выбрать так, чтобы оно делилось на $n$ и порядок $\bar{\varphi}_{c}$-орбиты элемента $b+B_{m}$ совпадал с порядком автоморфизма $\bar{\varphi}_{c}$, то $Z_{c}$ будет уже Р $\Phi$ А $\varphi_{c}$-группой, что и требуется.

Представим элементы $a$ и $b$ в виде $a=u(c), b=v(c)$ для некоторых многочленов $u, v \in \mathbb{Z}[x]$ и выберем число $\xi \in \mathbb{N}$ таким образом, чтобы числа $\left|u\left(\lambda_{j}\right)\right|$ и $\left|v\left(\lambda_{j}\right)\right|$ были меньше $\xi$ при каждом $j, 1 \leqslant j \leqslant q$. Далее воспользуемся леммой 3 и найдем число $m$, соответствующее $n$ и $\xi$.

Применяя теперь утверждение из формулировки леммы к парам многочленов $(u, v)$, $(v, u)$ и $(v, v)$, получаем, что при $0 \leqslant k \leqslant m / 2$ числа $N\left(a-c^{k} b\right), N\left(b-c^{k} a\right)$ и $N\left(b-c^{k} b\right)$ строго меньше $N\left(c^{m}-1\right)$. Поскольку для каждого ненулевого элемента $d \in Z_{c}$

$$
N\left(d\left(c^{m}-1\right)\right)=N(d) N\left(c^{m}-1\right) \geqslant N\left(c^{m}-1\right),
$$

это означает, что среди элементов $a-c^{k} b, b-c^{k} a$ и $b-c^{k} b, 0 \leqslant k \leqslant m / 2$, принадлежать подгрупше $B_{m}$ могут лишш те, которые равны нулю в группе $Z_{c}$.

Из условия предложения теперь сразу же следует, что $b-c^{k} b \notin B_{m}, 0<k \leqslant m / 2$,

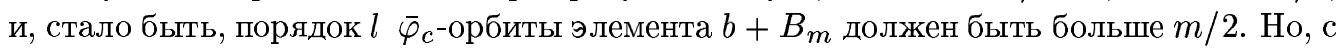
другой стороны, число $l$ делит порядок автоморфизма $\bar{\varphi}_{c}$, поэтому $l=m$.

Похожим образом проверяется и соотношение $a \nsim_{\varphi_{c}} b\left(\bmod B_{m}\right)$.

Так как $a \nsim_{\varphi_{c}} b$, то $a \neq c^{k} b$ и $b \neq c^{k} a$ при любом неотрицательном $k$. Поэтому элемент $a+B_{m} \in Z_{c} / B_{m}$ не принадлежит множеству $S=\left\{\left(b+B_{m}\right) \bar{\varphi}_{c}^{k} \mid-m / 2 \leqslant k \leqslant m / 2\right\}$. Но, как только что показано, порядок $\bar{\varphi}_{c}$-орбиты элемента $b+B_{m}$ равен $m$ и, значит, она полностью исчерпьвается элементами множества $S$. Таким образом, $a+B_{m} \nsim_{\bar{\varphi}_{c}} b+B_{m}$ и предложение, тем самым, доказано.

Следуюший шаг к решению рассматриваемой задачи позволяет сделать

ПРЕДЛОЖЕНИЕ 7. Пусть $A-$ свободная абелева группа конечного ранга $и \varphi-$ ее инбективный эндоморфизм. Если характеристический многочлен $\chi_{\varphi}$ әндомор-

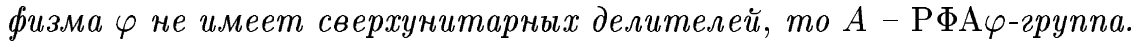

ДоКАЗАТЕЛЬСТво будем вести индукцией по рангу $r$ групшы $A$.

Если $r=1$, то $\chi_{\varphi}(x)=x-c$ для некоторого целого числа $c$, отличного от 0 и \pm 1 . Поэтому группа $A$ изоморфна $Z_{c}$ и при любом выборе изоморфизма $\rho: A \rightarrow Z_{c}$ эндоморфизмы $\varphi^{\rho}=\rho^{-1} \varphi \rho$ и $\varphi_{c}$ совпадают. Таким образом, в данном случае искомое утверждение вытекает из предложения 6.

Перейдем теперь к проверке индуктивного шага.

Пусть $V$ - некотороевекторное пространство над полем $\mathbb{C}$ размерности $r$. Мы можем, очевидно, считать $A$ подгруппой $V$, порожденной базисными векторами, а эндоморфизм $\varphi$ - сужением подходящего линейного оператора $\sigma$ пространства $V$. 
Пусть $v_{1}, v_{2}, \ldots, v_{r}$ - некоторьй жорданов базис оператора $\sigma$ и $V_{1}, V_{2}$ - подпространства пространства $V$, порожденные, соответственно, вектором $v_{1}$ и векторами $v_{2}, \ldots, v_{r}$. Рассмотрим два случая.

1) $A \cap V_{2} \neq 0$. Положим $A_{2}=A \cap V_{2}$. Очевидно, что подгруппа $A_{2}$ изолирована в группе $A$ и потому выделяется в ней прямым слагаемым. Обозначим через $A_{1}$ прямое дополнение этой подгрупшы до $A$.

Поскольку матрица $\sigma$ в базисе $v_{1}, v_{2}, \ldots, v_{r}$ является верхнетреугольной и невырожденной, $V_{2} \in \Omega_{\sigma}(V)$. Отсюда следует, что $A_{2} \in \Omega_{\varphi}(A)$ и мы можем определить ограничение $\varphi_{2}$ эндоморфизма $\varphi$ на подгрупп $A_{2}$ и эндоморфизм $\varphi_{1}$, индуцируемьй в группе $A_{1}$ отображением $\varphi$.

Пусть базис группы $A$ получен объединением некоторых базисов подгрупп $A_{1}$ и $A_{2}$, и пусть $M, M_{1}, M_{2}$ - матрицы эндоморфизмов $\varphi, \varphi_{1}, \varphi_{2}$ в этих базисах. Тогда ввиду $\varphi$-допустимости подгруппы $A_{2}$ матрица $M$ будет иметь следующий клеточньй вид:

$$
M=\left(\begin{array}{c|c}
M_{1} & * \\
\hline 0 & M_{2}
\end{array}\right),
$$

который говорит о том, что $\chi_{\varphi}=\chi_{\varphi_{1}} \cdot \chi_{\varphi_{2}}$ и, следовательно, характеристические многочлены $\chi_{\varphi_{1}}$ и $\chi_{\varphi_{2}}$ эндоморфизмов $\varphi_{1}$ и $\varphi_{2}$ также не имеют сверхунитарных делителей.

Поскольку группа $A$ содержит некоторый базис пространства $V$, она не может целиком лежать в подпространстве $V_{2}$. Стало быть, ранги подгрупп $A_{1}$ и $A_{2}$ строго меньше $r$ и мы можем применить к этим группам индуктивное предположение. Остается заметить, что группа $A_{1}$ изоморфна факторгрупе $A / A_{2}$ и с точностью до этого изоморфизма эндоморфизм $\varphi_{1}$ совпадает с эндоморфизмом $\varphi / A_{2}$. Таким образом, требуемое утверждение следует из второй части предложения 5.

2) $A \cap V_{2}=0$. В этом случае естественньй гомоморфизм пространства $V$ на факторпространство $\bar{V}=V / V_{2}$ определяет некоторое вложение $\rho$ групшы $A$ в $\bar{V}$. Так как матрица $\sigma$ в базисе $v_{1}, v_{2}, \ldots, v_{r}$ имеет нормальную жорданову форму, оператор $\sigma$ индуцирует в одномерном пространстве $\bar{V}$ некоторую гомотетию $\bar{\sigma}$ и коэффициент этой гомотетии равен одному из корней многочлена $\chi_{\varphi}$. С другой стороны, ограничение оператора $\bar{\sigma}$ на группу $A \rho$ совпадает, очевидно, с эндоморфизмом $\varphi^{\rho}=\rho^{-1} \varphi \rho$ данной группы.

Таким образом, учитьвая изоморфизм пространства $\bar{V}$ и поля комплексных чисел (рассматриваемого как векторное пространство над самим собой), мы можем считать далее группу $A$ подгруппой аддитивной групшы поля $\mathbb{C}$, а эндоморфизм $\varphi-$ ограничением отображения, умножающего каждьй элемент из $\mathbb{C}$ на некоторьй фиксированный корень $c$ многочлена $\chi_{\varphi}$. Отсюда, в частности, следует, что минимальный многочлен $g(x)=x^{n}-g_{n-1} x^{n-1}-\cdots-g_{1} x-g_{0}$ числа $c$ является также и минимальным аннулятором каждого элемента группы $A$.

Пусть $b \in A$ - произвольньй ненулевой элемент и $B$ - подгрупша, порожденная элементами $b \varphi^{i}, i \geqslant 0$. Очевидно, что подгруппа $B \varphi$-допустима, элементы $b, b \varphi, \ldots, b \varphi^{n-1}$, ввиду отмеченного только что свойства многочлена $g$, составляют ее базис, и матрица ограничения $\varphi_{B}$ эндоморфизма $\varphi$ на подгрупу $B$ имеет в этом базисе форму Фробениуса $(*)$. Но ту же форму имеет в базисе $c^{0}, c^{1}, \ldots, c^{n-1}$ и матрица эндоморфизма $\varphi_{c}$ группы $Z_{c}$. Кроме того, многочлен $g$ делит $\chi_{\varphi}$ и по условию не является сверхунитарным. Поэтому из предложения 6 следует, что $B$ является $Р \Phi А \varphi_{B}$-групшой. 
Обозначим через $A_{2}$ изолятор подгрупшы $B$ в группе $A$. Если $A_{2}=A$, то в силу второй части предложения $3 A$ также будет $\mathrm{P} \Phi А \varphi$-группй, и рассуждение на этом закончено. Поэтому далеемы будем предполагать, что подгруппа $A_{2}$ отлична от всей группы $A$.

Покажем, что $A_{2} \in \Omega_{\varphi}(A)$.

В самом деле, если $a$ - произвольньй элемент подгрупшы $A_{2}$, то для некоторого натурального числа $m m a \in B$. Но подгруппа $B \varphi$-допустима, поэтому $m(a \varphi)=(m a) \varphi \in B$ и, следовательно, $a \varphi \in A_{2}$.

Проверка $\varphi$-изолированности оказьвается несколько длиннее.

Если $a \varphi \in A_{2}$, то, как и вьше, найдется такое натуральное число $m$, что $m(a \varphi) \in B$. Запишем элемент тач в виде $\operatorname{ma\varphi }=m_{1} b+m_{2} b \varphi+\cdots+m_{n} b \varphi^{n-1}$.

Если $m_{1}=0$, то ввиду инъективности эндоморфизма $\varphi m a=m_{2} b+\cdots+m_{n} b \varphi^{n-2}$ $\in B$ и $a \in A_{2}$, что и требовалось. Поэтому далее мы можем считать, что $m_{1} \neq 0$.

Пусть $d=m a-m_{2} b-\cdots-m_{n} b \varphi^{n-2}$ и $D-$ подгрупша, порожденная элементами $d \varphi^{i}, i \geqslant 0$. Из соотношения $d \varphi=m_{1} b$ следует, что $d \neq 0$ и $m_{1} B \leqslant D$. Но ранги подгруп $m_{1} B$ и $D$ ввиду доказанного выше совпадают, поэтому порядок элемента $d$ по модулю подгрупшы $B$ конечен. Отсюда вытекает, что $d \in A_{2}, m a \in A_{2}$ и, так как подгруппа $A_{2}$ изолирована, $a \in A_{2}$.

Тем самым, подгрупша $A_{2}$ оказывается $\varphi$-изолированной, и рассуждение теперь завершается так же, как и в случае 1$)$.

Докажем, наконец, последнее утверждение, равносильное основной теореме ввиду предложения 1.

ПРЕДЛОЖЕНИЕ 8. Произвольная конечно порожденная абелева группа $A$ финитно аппроксимируема относительно ч-эквивалентности для каждого ее инбективного әндоморфизма

ДокАЗАТЕЛЬСТво. В силу предложения 2 и первой части предложения 5 нам достаточно найти такую подгруппу $B \in \Omega_{\varphi}(A)$, что ограничение $\varphi_{B}$ эндоморфизма $\varphi$ на эту подгрупшу является автоморфизмом, а факторгрупша $\bar{A}=A / B$ обладает свойством $\mathrm{P} \Phi \mathrm{A} \bar{\varphi}$, где $\bar{\varphi}=\varphi / B$.

Предположим сначала, что групша $A$ не имеет кручения.

Существование искомой подгруппы очевидно, если в некотором базисегрупшы $A$ матрица $M$ эндоморфизма $\varphi$ имеет вид (**), причем у характеристического многочлена матрицы $M_{1}$ нет сверхунитарных делителей, а характеристический многочлен матрицы $M_{2}$ является сверхунитарным. Подгруппа $B$ здесь порождается базисными элементами, соответствующими клетке $M_{2}$, а требуемые ее свойства вытекают из формы матрицы $M$, предложения 7 и замечания, сделанного в начале раздела.

В общем случае, как и при доказательстве предыдущего предложения, мы можем погрузить группу $A$ в векторноепространство $V$ соответствующей размерности, но уже над полем $\mathbb{Q}$, и считать эндоморфизм $\varphi$ сужением подходящего линейного оператора $\sigma$ пространства $V$.

Пусть $c_{1}, c_{2}, \ldots, c_{r}$ - какой-нибудь базис пространства $V$, в котором матрица $L$ оператора $\sigma$ имеет нормальную форму Фробениуса (см., например, [14, гл. VII, $\S 5]$ ). Более 
точно это означает, что $L$ - клеточно диагональная матрища:

$$
L=\left(\begin{array}{cccc}
L_{1} & & & 0 \\
& L_{2} & & \\
& & \ddots & \\
0 & & & L_{k}
\end{array}\right)
$$

каждая клетка $L_{i}$ которой имеет вид $(*)$ и характеристический многочлен, равный степени некоторого неприводимого над полем $\mathbb{Q}$ многочлена $f_{i}$.

Умножая при необходимости векторы $c_{1}, c_{2}, \ldots, c_{r}$ на одно и то же целое число, можем считать, что все они лежат в группе $A$ и, стало быть, порождают в ней некоторую подгруппу $C$ конечного индекса. Поскольку многочлены $f_{i}$ унитарны и являются, очевидно, делителями характеристического многочлена $\chi_{\varphi}$ эндоморфизма $\varphi$, они принадлежат кольцу $\mathbb{Z}[x]$. Поэтому матрица $L$ целочисленна и подгруппа $C \sigma$ - и $\varphi$-допустима.

Обозначая через $\psi$ ограничение эндоморфизма $\varphi$ на подгруппу $C$, видим, что матрица этого ограничения в базисе $c_{1}, c_{2}, \ldots, c_{r}$ совпадает с $L$ и что, перенумеровав подходящим образом базисные элементы, ее можно привести к виду, о котором шла речь в отмеченном вьше частном случае. Поэтому группа $C$ обладает такой подгруппой $D \in \Omega_{\psi}(C)$, что $\psi_{D} \in \operatorname{Aut}(D)$ и $\bar{C}=C / D-\mathrm{P} \Phi A \bar{\psi}$-группа (здесь, как обычно, $\psi_{D}=\left.\psi\right|_{D}$ и $\left.\bar{\psi}=\psi / D\right)$.

Пусть $B$ обозначает изолятор подгруппы $D$ в групше $A$. Как и в предыдущем предложении, легко проверяется, что подгруппа $B \varphi$-допустима, поэтому мы можем говорить об ограничении $\varphi_{B}$ эндоморфизма $\varphi$ на эту подгруппу. Покажем, что отображение $\varphi_{B}$ обратимо.

В самом деле, пусть $b$ - произвольный элемент группы $B$.

Поскольку подгруппа $D \quad \psi$-инвариантна и отображение $\psi_{D}$ является сужением эндоморфизма $\varphi_{B}$, последний индуцирует в факторгрупе $\bar{B}=B / D$ инъективньй эндоморфизм $\bar{\varphi}_{B}=\varphi_{B} / D$. Но группа $\bar{B}$ конечна; следовательно, отображение $\bar{\varphi}_{B}$ является автоморфизмом и сушествует такой элемент $b_{1}+D$, что $b+D=\left(b_{1}+D\right) \bar{\varphi}_{B}$.

Возврашаясь к групе $B$, получаем, что $b=b_{1} \varphi_{B}+d$ для некоторого элемента $d \in D$ и в силу уже упоминавшейся $\psi$-инвариантности подгрупшы $D d=d_{1} \psi_{D}$ для некоторого $d_{1} \in D$. Стало быть, $b=b_{1} \varphi_{B}+d_{1} \psi_{D}=\left(b_{1}+d_{1}\right) \varphi_{B}$, что и требовалось.

Таким образом, отображение $\varphi_{B}$ является автоморфизмом группы $B$ и, в частности, $B \in \Omega_{\varphi}(A)$. Остается лишь проверить, что факторгрупша $\bar{A}=A / B$ обладает свойством $\mathrm{P} \Phi \mathrm{A} \bar{\varphi}$.

Так как факторгрупа $\bar{C}=C / D$ является $\mathrm{P} \Phi \mathrm{A} \bar{\psi}$-группой, она не имеет кручения. Поэтому $C \cap B=D$ и $C / D=C / C \cap B \cong(C+B) / B$. Легко видеть также, что этот изоморфизм переводит эндоморфизм $\bar{\psi}$ групшы $\bar{C}$ в ограничение эндоморфизма $\bar{\varphi}$ на подгруппу $(C+B) / B$. Стало быть, группа $\bar{A}=A / B$ оказьвается расширением $\mathrm{P} \Phi$ А $\bar{\varphi}$-групшы $(C+B) / B$ и $|(A / B) /((C+B) / B)|=|A /(C+B)| \leqslant|A / C|<\infty$, так что искомое утверждение вытекает из второй части предложения 3.

Обратимся теперь к общей ситуации, когда $A$ является произвольной конечно порожденной абелевой групой, и рассмотрим факторгруппу $C$ групшы $A$ по ее периодической части $T$.

Поскольку подгрупа $T$, очевидно, $\varphi$-инвариантна, эндоморфизм $\varphi$ индуцирует в группе $C$ инъективньй эндоморфизм $\psi=\varphi / T$. Воспользуемся доказанным ранее и найдем такую подгруппу $D \in \Omega_{\psi}(C)$, что $\psi_{D} \in \operatorname{Aut}(D)$ и $\bar{C}=C / D$ является РФА $\bar{\psi}$-группой. 
Как и выше, проверяется, что подгруппа $B=D+T \varphi$-инвариантна, поэтому мы можем определить эндоморфизм $\bar{\varphi}=\varphi / B$ факторгруппы $\bar{A}=A / B$. Для завершения доказательства теперь остается заметить, что $\bar{A} \cong \bar{C}$ и с точностью до этого изоморфизма $\bar{\varphi}=\bar{\psi}$. Таким образом, $\bar{A}$ является $\mathrm{P} \Phi А \bar{\varphi}$-группой.

\section{СПИСОК ЦИТИРОВАННОЙ ЛИТЕРАТУРЫ}

[1] Raptis E., Varsos D. The residual finiteness of HNN-extensions and generalized free products of nilpotent groups: A characterization // J. Aust. Math. Soc. Ser. A. 1992. V. 53. № 3. P. 408-420.

[2] Raptis E., Talelli O., Varsos D. On the conjugacy separability of certain graphs of groups // J. Algebra. 1998. V. 199. № 1. P. 327-336.

[3] Молдаванский Д. И. Финитная аппроксимируемость некоторых HNN-расшшрений групп // Вестн. Иван. гос. ун-та. 2002. № 3. С. 123-133.

[4] Сенкевич О.Е. Финитная аппроксимируемость относительно сопряженности некоторых HNN-расширений групп // Алгебра и теория чисел. Современные проблемы и приложения. Тезисы докл. V Международной конференции (Тула, 19-24 мая 2003 г.). Тула: ТГПУ, 2003. C. 201-202.

[5] Молдаванский Д.И. Финитная аппроксимируемость нисходящих HNN-pacширений групп // Укр. матем. ж. 1992. Т. 44. С. 842-845.

[6] Rhemtulla A.H., Shirvani M. The residual finiteness of ascending HNN-extensions of certain soluble groups // Illinois J. Math. 2003. V. 47. № 1-2. P. 477-484.

[7] Каргаполов М.И., Тимошенко Е. И. К вопросу о финитной аппроксимируемости относительно сопряженности метабелевых групп // 4-й Всесоюзный симпозиум по теории групп. Тезисы докл. (Новосибирск, 5-9 февраля 1973 г.). Новосибирск, 1973. С. 86-88.

[8] Ремесленников В.Н. Сопряженность в полициклических группах // Алгебра и логика. 1969. T. 8. C. $712-725$.

[9] Сенкевич О.Е. О финитной аппроксимируемости относительно сопряженности нисходящих HNN-расширений конечно порожденных абелевых групп // Чебышевский сб. 2004. Т. 5. № 2. C. 121-130.

[10] Молдаванский Д. И., Кравченко Н. В., Фролова Е. Н. Финитная аппроксимируемость относительно сопряженности некоторых групп с одним определяюшим соотношением // Алгоритмические проблемы теории групп и полугрупп. Тула, 1986. С. 81-91.

[11] Мальцев А. И. О гомоморфизмах на конечные группы // Учен. зап. Иван. гос. пед. ин-та. 1958. T. 18. C. $49-60$.

[12] Носков Г. А. О сопряженности в метабелевых группах // Матем. заметки. 1982. Т. 31. № 4. C. 495-507.

[13] Линдон Р., Шупп П. Комбинаторная теория групп. М.: Мир, 1980.

[14] Гантмахер Ф.Р. Теория матриц. М.: Наука, 1966.

Ивановский государственный университет

Поступило

E-mail: ev-sokolov@yandex.ru

23.09 .2004

Исправленный вариант

28.03.2005 